\title{
Duplication 2 (q11.2-q21): a previously unreported abnormality
}

\author{
L B Cooke, H Richards, P W Lunt, L Burvill-Holmes, R T Howell, A McDermott
}

\begin{abstract}
A 7 year old boy is described with moderate learning disability, facial dysmorphism, and a de novo duplication of chromosome 2 (q11.2-q21). There are few published reports of proximal $2 q$ duplication, and none reporting direct de novo duplication for this exact region.
\end{abstract}

(f Med Genet 1995;32:825-826)

Duplication of distal regions of the long arm of chromosome 2 is well described and usually arises from a balanced translocation or insertion in either parent. It is associated with a characteristic phenotype. ${ }^{12}$

In contrast, proximal duplications of $2 \mathrm{q}$ are rarely described. The first case was reported by Ying $e t a l^{3}$ in 1984 (dup 2q11.2-2q14.2). Here we report a de novo duplication of 2 (q11.2-q21) in a 7 year old boy. The phenotypic features overlap with those described in cases of distal 2q duplication, but microcephaly and growth retardation are absent. There are also marked behavioural characteristics, namely hyperphagia and hyperactivity.

\section{Case report}

The proband was born at term, following a pregnancy complicated by first trimester threatened abortion and hyperemesis. Birth weight was $3120 \mathrm{~g}$ (50th centile).

Breast feeding could not be successfully es-

Stoke Park Hospital, Stoke Lane, Stapleton, Bristol BS16 1QU, UK

L B Cooke

$\mathrm{H}$ Richards

\section{Clinical Genetics Service, \\ Institute of Child Health, Bristol Royal Hospital for Sick Children, \\ St Michael's Hill, Bristol BS1 8BJ, UK \\ P W Lunt}

South Western Regional Cytogenetics Centre, Southmead Hospital, Bristol BS10 5NB, UK L Burville-Holmes $\mathrm{R}$ T Howell A McDermott

Correspondence to: Dr Cooke.

Received 3 November 1994 Revised version accepted for publication 15th May 1995
On physical examination at the age of 7 head circumference was on the 75 th centile. Facial features included a flat occiput and low posterior hairline, a broad, flat, low nasal tablished, so artificial feeding ensued. Developmental abnormality was suspected at 2 months by the mother, who described him as responding poorly to others. He was referred to, and subsequently followed up by, a neuropaediatrician. The proband smiled at 4 months, sat unaided at 8 months, and crawled by 20 months. At 18 months he was on the 50th the 90 th centile for weight. He could stand and walk by 22 months. He was usually clean and dry by day at 3 years 6 months, but not reliably so by night. He developed little intelligible speech before 4 years despite normal hearing and socialisation. He has had two episodes of Proteus urinary infection, although imaging of the renal tract has not shown structural abnormality. At the age of 7 years 4 months his mental age was 5 years 4 months (Leiter International Performance Scale) (IQ 73) bridge, and prominent, low set, shell-like ears. There were slight epicanthic folds and left convergent squint. The thumbs and big toes were broad, with hypoplastic nails. There was mild clinodactyly of the little fingers, bilateral planovalgus foot deformities, and genu valgum (fig 1). Genital anomalies included patent left processus vaginalis and left hydrocele.

His mother describes marked hyperactivity and poor sleep, with persistent waking at $4 \mathrm{am}$. He showed poor danger awareness, displaying no road sense, or fear of fire or water. Hyperphagia was a prominent feature, and he would eat until he vomited. Vomiting was sometimes self-induced. Food restriction was required to limit weight gain.

$\mathrm{He}$ was not compliant with formal psychological testing, showing poor concentration and motor restlessness.

By the age of 7 years the proband was able to dress himself and use a knife and fork. He could use the toilet if reminded. $\mathrm{He}$ enjoyed imaginative games and drawing but was boisterous and destructive. Speech was repetitive with a variable stutter.

Parental ages at birth were 20 years (mother) and 41 years (father). There is a paternal history of hypertrophic obstructive cardiomyopathy, although this has not been seen in the proband or his younger male sib, who is showing normal development.

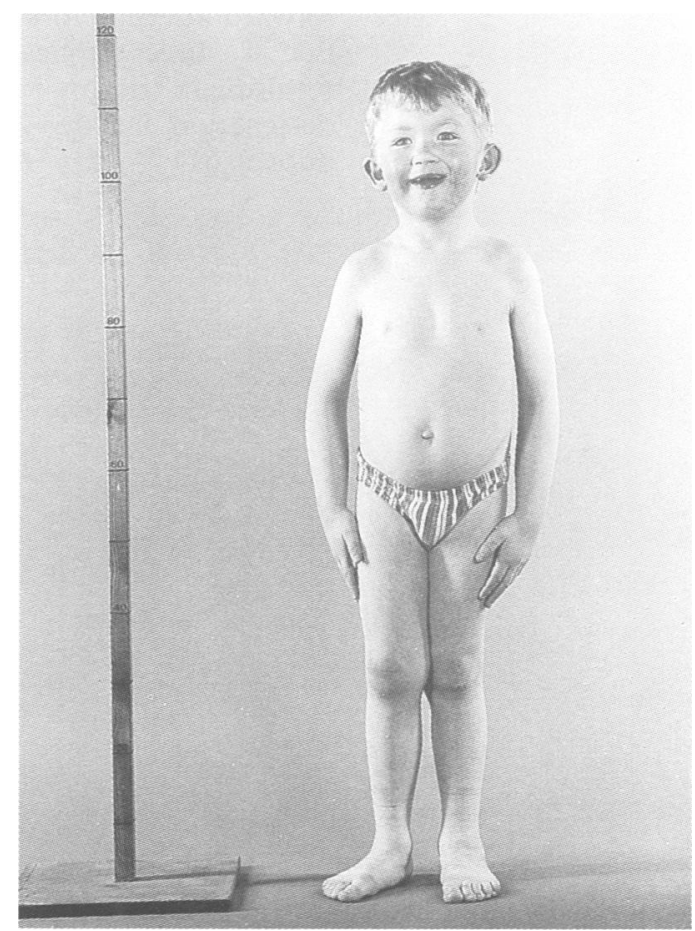

Figure 1 The proband aged 7 years. 


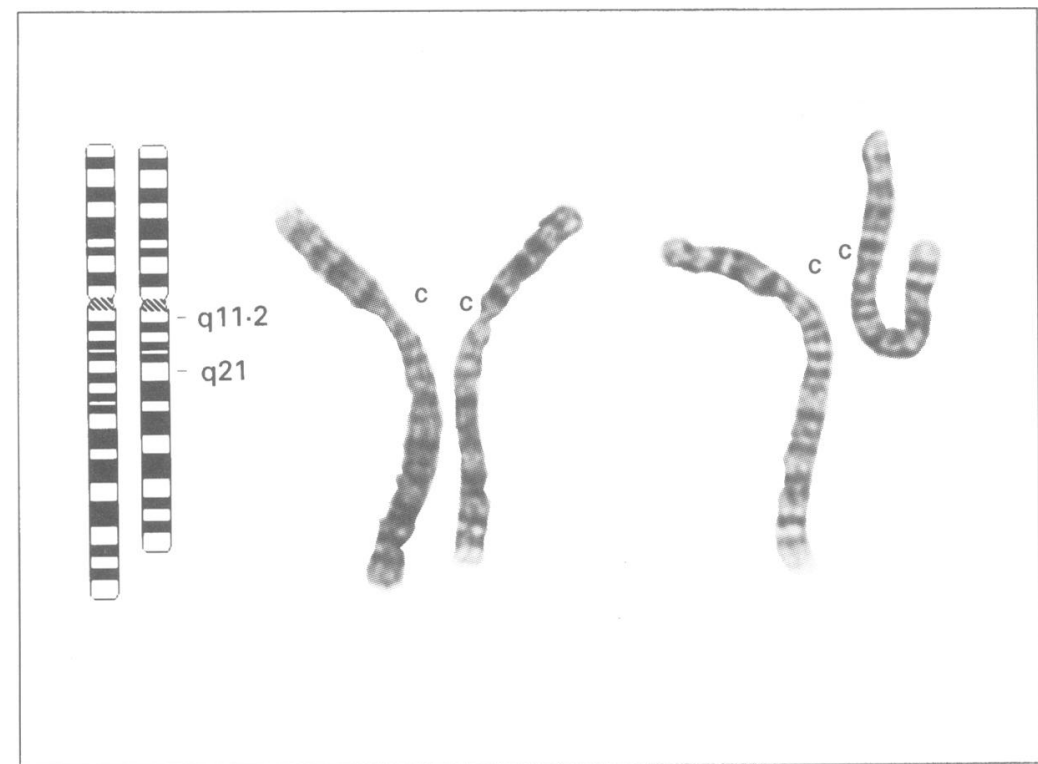

Figure $2 G$ banded homologues of chromosome 2 showing the duplication of bands q11.2-q21. The normal chromosome is on the right.

\section{CYTOGENETIC STUDIES}

Chromosome analysis was performed on preparations from synchronised lymphocyte cultures, stained using a standard trypsin/ Leishman protocol. An increase in the length of one homologue of chromosome 2 was observed, resulting from the presence of three extra small dark bands in the proximal part of the long arm (fig 2). In situ hybridisation using a chromosome 2 paint (Oncor, Alpha Laboratories, Eastleigh, UK) confirmed the extra material to have originated from chromosome 2, the whole of the abnormal chromosome displaying a positive fluorescent signal.

With reference to the banding pattern of the abnormal chromosome 2 , the most straightforward interpretation of the rearrangement is that of a direct duplication of bands q11.2-q21, resulting in trisomy for this region. The ISCN designation for this interpretation is $46, \mathrm{XY}$, $\operatorname{dup}(2)(\mathrm{q} 11.2-\mathrm{q} 21)$. As is the case with many de novo unbalanced abnormalities, other more complex explanations requiring multiple breakpoints cannot be completely ruled out.

Parental chromosomes were normal, as expected in the case of an abnormality such as this.

\section{Discussion}

Duplication of the proximal long arm of chromosome 2 is rarely reported. Our case shares some of the features reported by Ying et $a l^{3}$ namely developmental delay, depressed nasal bridge, and clinodactyly, but does not show congenital glaucoma. There is no microcephaly or growth retardation, common findings in aneuploidy syndromes.

The proband also shows features overlapping with those described in duplications of the distal long arm of chromosome 2 . The clinical syndrome of $\operatorname{dup}(2)$ (q35-2qter) is well recognised. ${ }^{12}$ The classical phenotype includes mental retardation, facial dysmorphism, and minor limb and external genital anomalies.

Behavioural aspects are infrequently mentioned in published reports and so comparisons are not possible. Our case was notable for sustained hyperactivity and hyperphagia. A concurrent diagnosis of Prader-Willi syndrome has been considered, but has been ruled out to date, given the absence of such features as hypotonia at birth, microcephaly, short stature, or a deletion abnormality of chromosome 15 .

We believe this to be the first reported case of dup (2) (q11.2-q21). Features include moderate learning disability and mild facial, limb, and external genital abnormalities, without growth retardation or microcephaly. This case also displayed hyperphagia and hyperactive behaviour.

1 Zankl M, Schwanitz G, Schmid P, et al. Distal 2q duplication: report of two cases and an attempt to define a syndrome. Am f Med Genet 1979;4:5-16.

2 Kyllerman M, Wahlstrom J, Westerberg B, Gustavson KH. Delineation of a characteristic phenotype in distal trisomy Delineation of a characteristic phenotype
2q. Helv Paediatr Acta 1984;39:499-508.

3 Ying $M$, Van Dyke DL, Weiss L, Olgac S. De novo direct Ying $M$, Van Dyke DL, Weiss $L$, Olgac $S$. De novo direct
tandem duplication of the proximal long arm of chrotandem duplication of the proximal long arm of chro-
mosome $2: 46, \mathrm{XX}$ dir $\operatorname{dup}(2)(\mathrm{q} 11.2 \mathrm{q} 14.2) . \mathcal{F}$ Med Genet 1984;21:57-8. 